\title{
Thygeson's Superficial Punctate Keratitis- Case Report and Review
}

\author{
S. K. Prabhakar \\ Department of Ophthalmology, J.S.S. Medical College \& Hospital/JSS University, India
}

\begin{abstract}
Thygeson's superficial punctate keratitis is a subtle, chronic and bilateral condition of the corneal epithelium, typically manifests with acute exacerbations and remissions. The etiology remains uncertain, despite the possibility of viral etiology was suspected. Topical steroid administration is useful in aborting an acute phase; but steroid induced cataract and glaucoma developed on long term usage. Topical cyclosporine A was used successfully for prolonged periods with minimal ocular surface irritation. The purpose of this case study was to highlight the importance of diagnosing the problem clinically and also to report efficacy and ocular side effects of topical cyclosporine A. Twenty six year old boy presented with persistent foreign body sensation in July 2011, since one month in both the eyes. Slit lamp microscopic examination revealed bilateral, multiple and punctate corneal epithelial lesions. The lesions resolved on topical steroids but recurred after cessation of the therapy. With the daily instillation of topical cyclosporine A, recurrence was not observed in two year follow up. Although topical steroid was useful in aborting an acute phase, the disease remitted on cessation of the therapy. Topical cyclosporine A, used for two years was effective in controlling the exacerbations without discernible ocular side effects.
\end{abstract}

Keywords: Cyclosporine A (CSA), Polymerase chain reaction (PCR), Thygeson's superficial punctate keratitis (TSPK)

\section{Introduction}

1.1 Dr Philips Thygeson described the problem first in the year 1950 and reported a case, characterized by bilateral corneal epithelial opacities with minimal or no conjunctival congestion without the stromal association attributable to virus particles. ${ }^{[1,2]}$ The reason for the problem was investigated, but obtained negative proofs of virus by tissue culture and Electron microscopy. Polymerase chain reaction by material amplification also failed to demonstrate that the disease could be of viral in origin ${ }^{[3]}$ Varicella Zoster virus genome could not be detected by PCR in any of the patients, and although in one case the virus detected from the corneal lesions. ${ }^{[4]}$ There was an association of histocompatible lymphocyte antigen (HLA) $\mathrm{DR}_{3}$ as revealed by the previous studies. ${ }^{[5]}$ Despite the prolonged and protracted course of the disease, visual prognosis was excellent even after ten years of follow up. ${ }^{[6]}$

1.2 Thygeson's superficial punctate keratitis is essentially an objective opinion with other investigations proven to be discouraging. The disease manifests bilaterally with irritation, photophobia, excessive watering, and foreign body sensation and on occasions, with visual disturbances in the acute phase. The purpose of presenting the present case report is to highlight the importance of diagnosing the disease clinically by excluding the other causes that produce punctate epithelial lesions. And also to find out the efficacy of cyclosporine A on long term usage and its complications. The clinical findings and management in this section were correlated and discussed with the currently available literature.

\section{Case History}

2.1 A twenty six year old male patient presented with chronic foreign body sensation and photophobia in both the eyes for the last one month in the year July 2011. The patient was apparently normal previously before the development of the symptoms although some self-limiting discomfort was noticed. On routine physical examination, the young boy was otherwise healthy and fairly built. Anterior segment examination revealed 9 to12 multiple, grayish-white epithelial lesions distributed mainly in the central cornea of both the eyes with intact corneal sensation (Fig 1 and 2). Few of the lesions stained with fluorescein and others had mild staining. With minus one spherical lens, the best corrected visual acuity was 20/20 with normal near vision. Schirmer's test for tear fluid secretion showed a wetting of the tear strip about $14 \mathrm{~mm}$ and $15 \mathrm{~mm}$ in the right and left eyes respectively. Anterior chamber and dilated fundoscopy was unremarkable. The lid margins and the ocular adnexa were normal on slit lamp biomicroscope examination.

2.2 Considering the classical symptoms and clinical presentation a clinical diagnosis of TSPK was made. Artificial eye drops with antibiotic and steroid compound (Gatifloxicin $0.3 \%$ plus dexamethasone $0.3 \%$ ) four times a day for a week was recommended. Education regarding the condition made clear to the patient and advised to respond immediately on any increase in the symptoms. There was a remarkable reduction of 
symptoms with resolution of most of the lesions. The topical steroid tapered in a measure of 3 times for two weeks, 2 times for two weeks and once a day for two weeks. The patient was asymptomatic during the application period but only to return to the Hospital with the appearance of the same symptoms and white punctate corneal lesions as before. Then $2 \%$ topical Cyclosporine A eye drops prescribed two times a day for one month. Once again he presented with the same complaints, reporting that the symptoms and signs have appeared on termination of the drug. We advised the patient to use topical CSA continuously once or twice a day to control the symptoms. After about two years, the patient is still using the cyclosporine A eye drops, and pleased that the disease has not recurred.

\section{Results}

3.1 Punctate corneal epithelial lesions resolved on topical application of steroids initially followed by recurrence of similar episodes on stoppage of the steroids. Topical treatment with Cyclosporine A showed excellent results in controlling the recurrence in addition to improvement in subjective signs and symptoms.

\section{Discussion}

4.1 The exact cause of TSPK is unclear in that a viral infection suspected and failed to establish genomes by PCR amplification against Varicella Zoster, Herpes Simplex and other viruses. This suggested that the disease might be due to an autoimmune process that induces inflammatory response in the corneal epithelium. TSPK is mostly a clinical diagnosis based on the presence of corneal physical signs. In the present case scenario herpes simplex keratitis was excluded by the absence of typical dendrite pattern and corneal anesthesia. Herpes zoster keratitis manifests mainly as cutaneous lesions distributed across the trigeminal nerve dermatomes with its classical presentation was also not considered. Other problems that produce superficial epithelial keratitis like posterior blepharitis, rosacea keratitis and drug induced were ruled out.

4.2 Increased Langerhans cells in the basal cell layer was demonstrated by in vivo laser confocal microscopic study of changes in the corneal epithelium, hypothesized the role of inflammation as possible pathogenesis of the disease. ${ }^{[7]}$ Nevertheless the problem is tractable for and extremely receptive to immunemodulator agents such as steroids or cyclosporine. Although in the present case, an acute attack was aborted with topical steroids, but the symptoms reverted on termination of the medication. With long term topical administration, steroids might cause bilateral posterior sub capsular cataracts and induced glaucoma in steroidresponders. Probably a locally activated immunological response might be checked by instillation of cyclosporine-A eye drops.

4.3 CSA is a powerful immune-modulator, indicated in keratoconjunctivitis sicca, vernal keratoconjunctivitis, superior limbic keratoconjunctivitis and atopic conjunctivitis as a topical application. In the previous case report, CSA was used for four and half years without any ocular surface problems except for mild stingy sensation. Topical antiviral agents are inadequate or might cause epithelial toxicity than promoting the healing of the lesions. The situations where the steroids drops were to be ineffective or signs of emergence of ocular side effects observed topical cyclosporine is the best alternative treatment even for prolonged course of therapy. The use of topical CSA as a primary modality of treatment was recommended by the previous report. ${ }^{[8]}$ Use of artificial tear drops as an adjuvant and soft bandage contact lenses in extreme severe cases alleviated not only the symptoms but also helps in rapid promotion of the healing. ${ }^{[9]}$

\section{Conclusion}

5.1 TSPK is a condition of unknown etiology that can easily be diagnosed by clinical examination alone which responds directly to topical steroids. In the present case scenario, patient had been benefited by the instillation of topical cyclosporine A with minimal stingy feeling of the eyes. Recurrences of the epithelial lesion were not observed in two year follow up. Further research may be extended to the assessment of the immunological status of patients to find out whether the disease is a localized manifestation of an autoimmune phenomenon.

\section{References}

[1]. THYGESON P (1950). Superficial keratitis. J Am Med Assoc; 144(18):1544-9.

[2]. Duszak RS (2007). Diagnosis and management of Thygeson's superficial punctate keratitis. Optometry; 78(7):333-8.

[3]. Connell PP et al (2007). The role of common viral ocular pathogens in Thygeson's superficial punctate keratitis. Br J Ophthalmol; 91(8):1038-41.

[4]. Reinhard T et al (2004). PCR for varicella zoster virus genome negative in corneal epithelial cells of patients with Thygeson's superficial punctate keratitis. Eye (Lond); 18(3):304-5.

[5]. Darrell RW (1981). Thygeson's superficial punctate keratitis: natural history and association with HLA DR3. Trans Am Ophthalmol Soc; 79:486-516.

[6]. Nagra PK et al (2004). Thygeson's superficial punctate keratitis: ten years' experience. Ophthalmology; 111(1):34-7.

[7]. Kawamoto K et al (2009). In vivo observation of Langerhans cells by laser confocal microscopy in Thygeson's superficial punctate keratitis. Mol Vis; 15:1456-62. 
[8]. Hasanreisoglu M (2008). Long-term topical cyclosporin A therapy in Thygeson's superficial punctate keratitis: a case report. Cases J; 1(1):415.

[9]. Goldberg DB et al (1980). Management of Thygeson's superficial punctate keratitis. Am J Ophthalmol; 89(1):22-4.

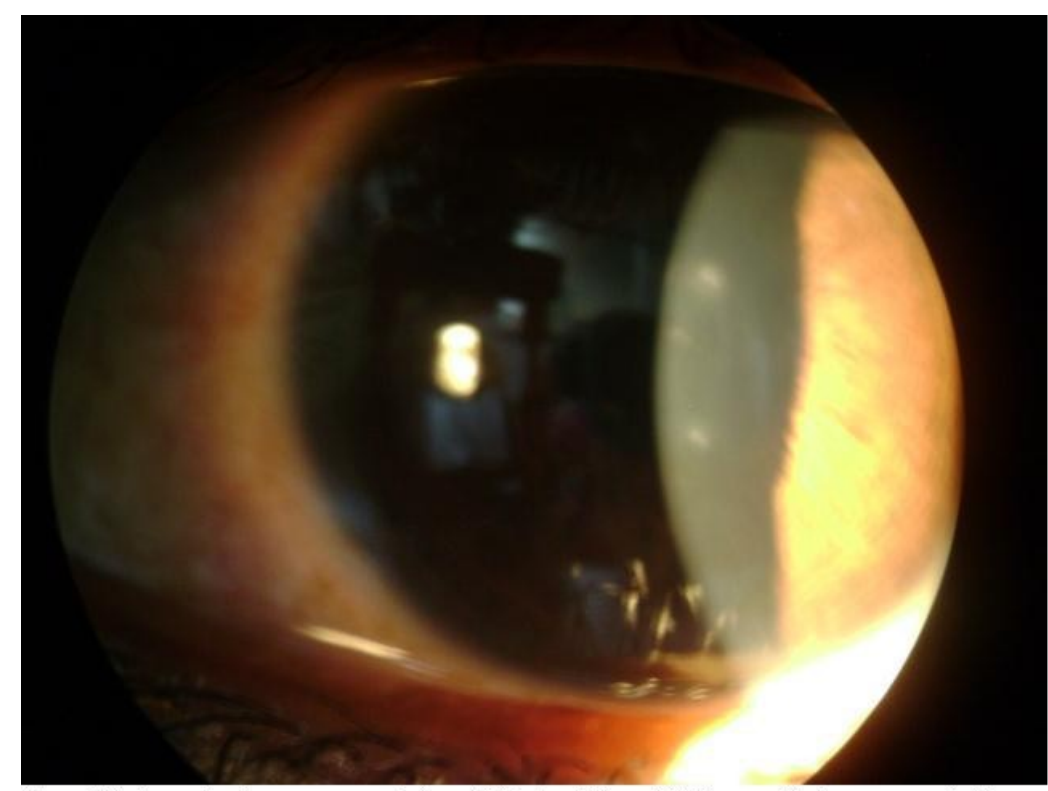

Figure2. Left eye showing coarse, punctate and ill-defined intraepithelial corneal lesions surrounded by halo of haziness.

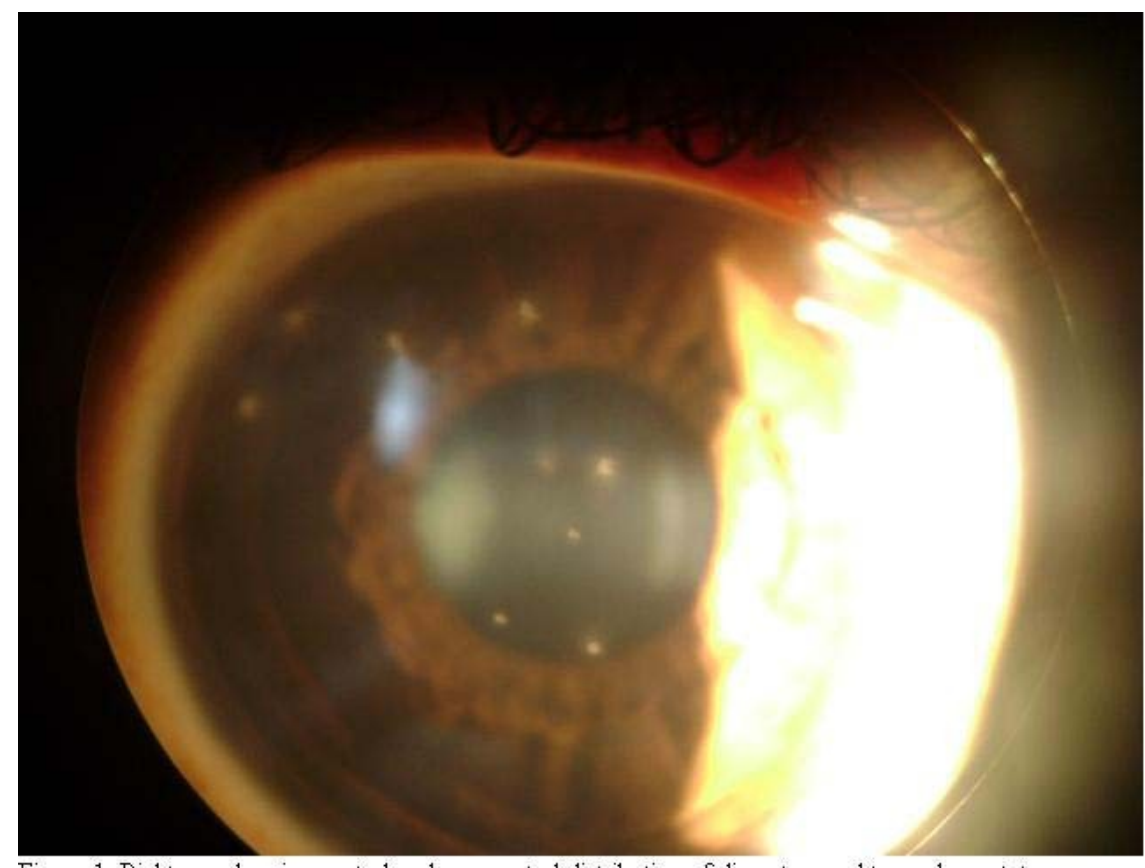

Figure 1. Right eye showing central and paracentral distribution of discret, round to oval punctate whitish-gray intraepithelial corneal lesions. 